
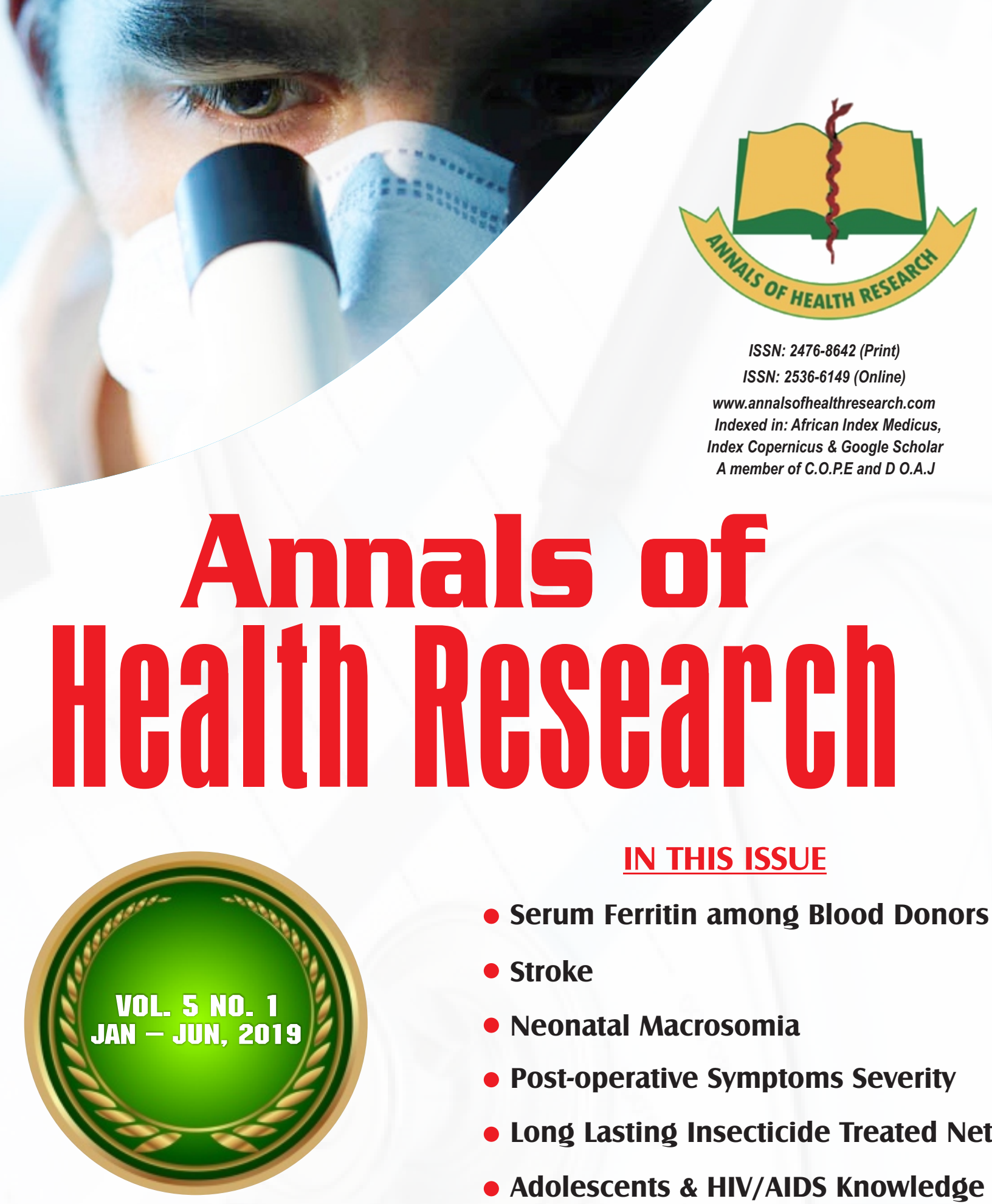

\title{
IN THIS ISSUE
}

- Serum Ferritin among Blood Donors

- Stroke

- Neonatal Macrosomia

- Post-operative Symptoms Severity

- Long Lasting Insecticide Treated Nets

- Adolescents \& HIV/AIDS Knowledge

- Emergency Obstetric Hysterectomy

- Lower Third Molar Surgery

- Thyroid Disorders

- Serum Vitamin D and Pneumonia

PUBLISHED BY THE MEDICAL 


\title{
A seven-year retrospective review of stroke admissions in Benin-City, Southern Nigeria Kayode-Iyasere $\mathrm{E}^{1}$, Odiase $\mathrm{FE}^{* 2}$ \\ ${ }^{1}$ Department of Medicine, Central Hospital, Benin City, Nigeria ${ }^{2}$ Department of Medicine, University of Benin Teaching Hospital, Benin City, Nigeria
}

\section{*Correspondence: Dr FE Odiase, Department of Medicine, University of Benin Teaching Hospital, Benin-City, Nigeria; Email: francisodiase2000@hotmail.com; ORCID: https://orcid.org/0000-0001-9788-6482}

\begin{abstract}
Background: Stroke is a significant cause of hospital admissions, disability and mortality, but there is a lack of information on stroke in parts of the southern region of Nigeria.

Objective: To analyze the frequency of stroke admissions, clinical presentations, risk factors, stroke types and outcomes over seven years, in a secondary level hospital in southern Nigeria.

Methods: This was a retrospective study. The hospital record of patients hospitalized between January 2006 and December 2012 at the Central Hospital, Benin-City with the diagnosis of stroke based on the World Health Organization (WHO)-clinical criteria, were studied.

Results: Four hundred and nineteen patients with stroke were hospitalized during the study period; this accounted for $3.1 \%$ of all the medical admissions. The mean age was $62.4 \pm 13.6$ years. There were $222(53 \%)$ males and $197(47.0 \%)$ females. Ischaemic stroke occurred among 71\% of cases; others included intra-cerebral haemorrhage $(26 \%)$ and subarachnoid haemorrhage (3.1\%). The main presenting features included hemiparesis $(69 \%)$ and sudden loss of consciousness (27.2\%). The risk factors included hypertension (84\%) and diabetes mellitus $(12.2 \%)$. The 7, 14 and 30 days case fatality rates were $21.2 \%, 25.5 \%$ and $30.8 \%$ respectively. A higher case fatality rate was recorded in haemorrhagic stroke compared to ischaemic stroke ( $68.8 \%$ vs $24 \%, \mathrm{p}=0.0001)$.

Conclusion: Stroke was a significant cause of mortality amongst medical admissions and hypertension was the most common risk factor. Closer attention to the risk factors for stroke may likely help to reduce the burden of stroke.
\end{abstract}

Keywords: Diabetes mellitus, Hypertension, Hemiparesis, Intra-cerebral haemorrhage, Ischaemic stroke, WHOClinical Criteria for stroke

\section{Introduction}

Stroke, as defined by the World Health Organization (WHO), refers to rapidly developing clinical signs of focal (or global) disturbance of cerebral function lasting more than 24 hours or leading to death with no apparent cause other than that of vascular origin. [1] The American Heart Association/ American Stroke Association (AHA/ASA)proposed a definition of stroke which incorporates clinical and tissue criteria as follows: brain, spinal cord, or retinal cell death 
attributed to ischaemia with pathological, imaging or other objective evidence of cerebral, spinal cord or retinal focal ischaemic injury in a defined vascular distribution or clinical evidence of cerebral, spinal or retinal focal ischaemic injury based on symptoms persisting for greater than 24 hours or until death and with other aetiologies excluded. [2] Stroke includes clinical signs and symptoms suggestive of subarachnoid haemorrhage, intracerebral haemorrhage or cerebral ischaemic necrosis, but excludes transient ischaemic attacks, subdural hematoma and haemorrhage or infarction caused by infection or tumour. [1 -3]

Globally, stroke continues to be a leading cause of hospital admissions, mortality and physical disability. About 18 million people worldwide suffer from stroke every year, nearly six million people die, and another six million people are left permanently disabled. [4] The degree of disability varies from mild clumsiness in the fingers of one arm to total paralysis of one arm or leg with or without loss of speech or swallow. [4]

By the WHO definition of stroke, the condition is diagnosed clinically, but laboratory investigations and brain imaging can complement the clinical diagnosis and are essential for many decisions regarding treatment. The diagnosis of stroke and, in particular, its subtypes currently relies on a confirmatory emergency Computerized Tomographic Scan of the brain. The diagnosis ensures immediate thrombolytic administration in cases of ischemic stroke, or endovascular thrombectomy and early aneurysmal clipping in subarachnoid haemorrhage (SAH). However, in settings where neuroimaging is not possible, the WHO Clinical Criteria for stroke can be utilized, and this becomes equally important for prompt diagnosis and treatment. The latter includes early stabilization of vital signs such as high blood pressure control, hydration, oxygen therapy and reversal of hyper/hypoglycaemia among others.

In developed societies, immediate emergency CT brain scan has been the rule for several years. Unfortunately, emergency computerized tomography of the brain following stroke is a challenge in many hospital settings (health centres, general hospitals, and state hospitals) in the developing world. Several reasons have been adduced for this limitation, including the lack of CT machines in many hospitals, poorly functional neuro-imaging units characterized by the frequent breakdown of CT machines, the prohibitive cost of neuro-imaging, availability of CT scan machine in few urban centres and poor knowledge of stroke. [5] Many, including the Central Hospital, Benin-City (the location of this study) relied, on the WHO Clinical Criteria for the diagnosis of stroke because of unavailability of CT scan. The WHO Clinical Criteria have been shown to have an overall accuracy of $71 \%$ among Nigerians with stroke. [7] Using this criteria, a patient is classified as having ischaemic stroke, if there is an acute onset of focal neurological deficit with or without impairment of consciousness, which may have been preceded by a transient ischemic attack, occurred at rest or with normal or mild elevation of the blood pressure, and there is no associated headache or vomiting and the cerebrospinal fluid is usually clear. Intracerebral haemorrhage typically occurs during activity with a rapidly changing neurological deficit and impairment of consciousness. There may be moderate to severe elevation of the blood pressure, and the cerebrospinal fluid may have been bloody; vomiting and headache are frequent, but previous transient ischemic attack is uncommon. Subarachnoid haemorrhage usually presents with sudden severe "thunderclap" headache, which is often accompanied by vomiting; there may be neck stiffness, and the cerebrospinal fluid may be bloody or xanthochromic. ${ }^{[6,7]}$ 
Few conditions can mimic stroke; these include seizures, conversion disorder, migraine headache and hypoglycaemia. These constitute a tiny percentage, and the clinical course of the illness can be ascertained by performing simple laboratory tests (such as blood sugar estimation) which often distinguishes these stroke-mimics from a vascular stroke. It has been found that when the diagnosis of stroke is based on adequately designed clinical criteria, the percentage of diagnostic errors in stroke is low. [9]

There is a lack of information with regards to stroke epidemiology in the southern region of Nigeria. The authors are not aware of any study that had described the pattern of stroke in this region of Nigeria using the WHO Clinical Criteria, especially when neuro-imaging facilities are not available. This present study, retrospective in design, was intended to assess stroke trends, prevalence, patterns, risk factors, the relationship between stroke types and outcomes and mortality of stroke admissions during seven years. This study would provide information on stroke data in the southern region of Nigeria and also serve as a basis for comparisons with future stroke studies involving neuro-imaging.

\section{Methods}

The study was a retrospective analysis of stroke admissions in the medical wards of Central Hospital, Benin-City, a secondary level hospital located in the city centre. It is the leading specialist state-owned hospital with a Consultant Neurologist in attendance. The Neurology Unit, using the WHO Clinical Criteria, identified all stroke admissions and entered the relevant data into a stroke registry.
Patients admitted between January 2006 and December 2012 with the diagnosis of stroke, based on the WHO Clinical Criteria, were enrolled in the study from the hospital's stroke registry. The case records of these patients were retrieved and reviewed in detail. The data extracted from the record included demographic parameters, stroke type, clinical features, risk factors, duration of hospitalization and outcome (whether the patient was appropriately discharged, discharged against medical advice (DAMA) or died in the course of admission). A data collection sheet was designed with the above-listed variables to facilitate the process. Also, available data regarding admissions into the hospital, admissions into the medical wards and emergency presentations throughout the study were also obtained from the hospital's record unit. The hospital's Ethics Committee approved the study.

The statistical analysis was performed using SPSS version 21. Continuous variables were presented using means, median, standard deviation and range and these were compared using the Student's t-test. Frequency and percentages were used to summarize categorical variables and Chi-Square test was used to assess the association between these variables. Statistical significance was set at $95 \%$ probability $(\mathrm{p}<0.05)$.

\section{Results}

During the 7-year period, a total of 67, 484 patients were admitted into the hospital; 13,589 of these were medical admissions while 7,740 were emergency admissions. There were 419 stroke admissions, representing $0.6 \%$ of total hospital admissions, $5.4 \%$ of emergency admissions and $3.1 \%$ of medical admissions. The mean age of the stroke patients was $62.4 \pm 13.6$ years, with an age range of 18 to 99 years. Two hundred and twenty-two (53.0\%) of the stroke 
patients were males while $197(47.0 \%)$ were females with a male to female ratio of 1.1: 1 . Stroke was the indication for admission of $3.5 \%$ of males and $2.7 \%$ females into the medical wards while, for the emergency admissions, stroke accounted for $4.7 \%$ and $6.6 \%$ of males and females respectively.

Patients with ischaemic stroke constituted 70.9\% $(279 / 419)$ of the cases, while $26.0 \%(109 / 419)$ and $3.1 \%(13 / 419)$ had intra-cerebral haemorrhage and subarachnoid haemorrhage, respectively. Clinical presentations included hemiparesis/ hemiplegia (69.0\%), sudden loss of consciousness $(27.2 \%)$, facioparesis $(2.4 \%)$, loss of balance/dizziness (0.7\%) and dysphasia (6.0\%). Eighty-four per cent (352) of stroke patients had hypertension, and it was the commonest risk factor identified. This was followed by diabetes mellitus (12\%), significant alcohol ingestion $(2.6 \%)$ and previous stroke $(2.4 \%)$ as shown in Table I.

The attending physician discharged Fifty-three per cent (222) of the stroke admissions, 37.2\% (156) died during the hospitalization while 9.8\% (41) of the cases were discharged against medical advice (DAMA). Half of the deaths from stroke occurred in the first four days of admission. Eighty-nine deaths occurred by day 7 of admission giving a case fatality rate of 21.2 $\%$. One hundred and seven deaths occurred by day 14 of admission, corresponding to a case fatality rate of $25.5 \%$ while $82.7 \%$ deaths occurred within 30 days of admission giving a 30 -day case fatality rate of $30.8 \%$. There was no significant difference among the mean ages of the three outcome groups. More patients with intra-cerebral haemorrhagic stroke compared to patients with ischaemic stroke died during the 7 -year period $(68.8 \%$ versus $24.0 \%$; $p=0.0001)$ as shown in Table II.

\section{Discussion}

This study has shown that over the 7-year study period, stroke was responsible for $3.1 \%$ of all medical admissions. This is similar to an earlier study by Ogun in Lagos University Teaching Hospital, which showed that $3.7 \%$ of medical admissions were due to stroke [10], but considerably lower when compared to $7.3 \%$ of medical admissions in Sokoto. [11] The present study showed stroke accounted for $5.4 \%$ of emergency admissions; this was high when compared to $2.4 \%$ recorded by Ogun et al. ${ }^{[12]}$ at the Ogun State University Teaching Hospital (OSUTH), Sagamu. The higher rate recorded in the present study could be attributed to the prompt response of patients' relatives to victims of stroke as a result of increased awareness and knowledge that stroke is an emergency. [13] The stroke admissions accounted for $0.6 \%$ of the total hospital admissions during the period under review, and this has been the pattern from other hospital-based studies in Nigeria. [11, $14,15]$

In the study, there was a slight male preponderance with a male to female ratio of 1.1:1. This is dissimilar to other studies which had earlier reported male dominance in stroke. $[11,16-18]$ and supports the fact that stroke is more common in males than females. Stroke incidence rates are 1.25 times greater in men, but because women tend to live longer than men, more women than men die of stroke each year. [19]

The mean age of the study population was similar to what had been described in earlier studies $^{17}$ in the same geopolitical zone and elsewhere, [20 - 22] but lower than the usual age of stroke in industrialized countries. [23, 24] It is possible that ignorance, poverty and lifestyle may play a role.

The distribution of stroke types in the present study revealed two distinct types of stroke. Clinical distinction between cerebral 
haemorrhage and infarction using the WHO definition, are, in no small extent precise and has been well documented. [7] A prodrome of headache, a feeling of awry in the head, cerebral seizures, vomiting with nuchal pain and rigidity at or soon after onset in addition to varying degrees of impaired consciousness are features suggestive of haemorrhagic stroke.

A history of acute onset of a focal neurological deficit with or without impairment of consciousness may be preceded by previous transient ischemic attack occurring at rest and "stuttering" progression of symptoms and signs while not invariably present, were strongly presumptive of cerebral infarction. The abruptness of symptoms observed in some cases in this study strongly suggested cerebral embolism. The finding of normal to mild blood pressure elevation with no associated history of headache and vomiting was also used in this study to support cerebral infarction, while the finding of moderate to severe blood pressure and bloody cerebrospinal fluid, where indicated, also strongly supported the haemorrhagic stroke. In this study, ischaemic stroke predominated with $71 \%$, compared to cerebral haemorrhagic stroke, which accounted for $26.0 \%$ and

$3.1 \%$

as

SAH.

Table I: Demographic characteristics and clinical features of study participants

\begin{tabular}{|c|c|c|c|}
\hline Clinical parameters & & Frequency & Percentage \\
\hline \multirow[t]{9}{*}{ Risk Factors } & Hypertension & 352 & 84.0 \\
\hline & Diabetes & 51 & 12.2 \\
\hline & Alcohol & 11 & 2.6 \\
\hline & Previous stroke & 10 & 2.4 \\
\hline & Smoking & 4 & 1.0 \\
\hline & Obesity & 2 & 0.5 \\
\hline & Chronic kidney disease & 1 & 0.2 \\
\hline & Heart failure & 1 & 0.2 \\
\hline & Transient ischemic attack & 1 & 0.2 \\
\hline \multirow[t]{7}{*}{ Multiple Risk Factors } & & 50 & 11.7 \\
\hline & Hypertension/ Diabetes mellitus & 37 & 8.8 \\
\hline & Hypertension/Alcohol & 6 & 1.4 \\
\hline & Hypertension/Smoking/Alcohol & 3 & 0.7 \\
\hline & Hypertension/Obesity & 2 & 0.5 \\
\hline & Hypertension/Previous stroke & 1 & 0.2 \\
\hline & Smoking/Alcohol & 1 & 0.2 \\
\hline \multicolumn{4}{|l|}{ Clinical Presentations } \\
\hline & Hemiparesis/Hemiplegia & 289 & 69.0 \\
\hline & Sudden loss of consciousness & 113 & 27.2 \\
\hline & Facioparesis & 10 & 2.4 \\
\hline & Dysphasia & 25 & 6.0 \\
\hline & Loss of balance/dizziness & 3 & 0.7 \\
\hline
\end{tabular}


Table II: The association between types of stroke and types of clinical outcomes

\begin{tabular}{llll}
\hline Variables & Outcomes & & \\
\hline & DAMA & Died & Discharged \\
\hline Frequency of Outcome (\%) & $41(9.8)$ & $156(37.2)$ & $222(53.0)$ \\
\hline Age yrs (Mean +SD) & $60.3 \pm 13.9$ & $62.5 \pm(12.9)$ & $62.8 \pm(14.0)$ \\
IC-Hemorrhagic n\% & $4(3.7)$ & $75(68.8)$ & $30(27.5) *$ \\
Ischemic n\% & $36(12.0)$ & $71(24.0)$ & $190(64.0) *$ \\
SAH n\% & $1(7.7)$ & $10(76.9)$ & $2(15.4) *$ \\
Duration of admission (days) & $3(1-7)$ & $4(1-9.5)$ & $7(4-15)$ \\
[Median (IQR)] & & & \\
\hline
\end{tabular}

KEY: * P < 0.0001; DAMA - Discharged against medical advice; SAH - Subarachnoid Haemorrhage; IC-hemorrhagic - Intracerebral Haemorrhage; IQR - Interquartile Range.

This pattern of stroke distribution is well established in the literature. [10, 16, 18, 22, 25] Various studies have given conflicting reports on the pattern of stroke type distribution; while some have reported a higher incidence of ischaemic stroke, [20,25, 26] other studies have documented a preponderance of haemorrhagic stroke. [12, 15] Reasons for this variance could be attributed to different parameters used in classifying stroke types; while some studies have used clinical distinction and Siriraj score which are considered less reliable by best practices, others have used, where available and affordable, Computerized Tomographic scan (CT Scan). Using the latter, separation of the two basic vascular processes, infarction and haemorrhage, is almost complete and thus, more reliable.

In the present study, hypertension and diabetes mellitus were the two most important modifiable risk factors for stroke $(84.2 \%$ and $12.2 \%$ respectively). This pattern was similar to the finding in the study done by Imariagbe et al., where the finding of hypertension was also remarkably high and closely followed by diabetes mellitus. [17] The finding of hypertension as a dominant risk factor for stroke agrees with reports from several studies carried out in Africa and the developed countries [14, 15, 20, 21, 25, 26] and calls for an urgent need for intervention. In this study, the median duration of admission was seven days. This is comparable to the mean duration of stay recorded by Ojo et al. [18] Of the 156 deaths recorded in the present study; half occurred within the first four days of admission while $82.7 \%$ occurred within 30 days of admission. This is not surprising as more than two-thirds of patients diagnosed with haemorrhagic stroke in this study died compared to $24.0 \%$ of patients diagnosed with ischaemic stroke. This observation supports the fact that haemorrhagic stroke was associated with a more unsatisfactory outcome with statistical significance. It is also noteworthy that $55.0 \%$ of stroke patients were discharged following recovery. This was similar to reports previously recorded from other studies. [18, 19] In this study, $9.8 \%$ of the patients were discharged against medical advice compared to $13.3 \%$ and $20.4 \%$ previously reported by Ojo et al ${ }^{[18\}}$ and Sennay et al ${ }^{20\}}$ Unmet expectation of immediate improvement by the relatives coupled with the strong belief in traditional and spiritual healing methods could be responsible for the high rate of DAMA.

There was no significant difference in the mean ages of patients distributed according to the three outcome types. In the present study, the 7- 
day and 30-day case fatality rates were $22.2 \%$ and $30.8 \%$ respectively; these were higher than the generally known 7-day and 30-day case fatality rates of $12 \%$ and $19 \%$ respectively [27] but comparable to reports from other studies in Sub-Saharan Africa. $[10,12,25,28,29]$

The limitations of this study included its retrospective design with no control of the researcher over the standards or comprehensiveness of the data retrieved. In addition, this study is based on WHO Clinical Classification; hence, a more extensive prospective study with neuroimaging confirmation is required for more detailed and accurate studies in the environment.

\section{Conclusion}

Stroke remains a major cause of death in this study. More than $50 \%$ of deaths occurred within four days. The high mortality rate due to haemorrhagic stroke is attributable to severe uncontrolled hypertension, which is the dominant risk factor in this study and calls for urgent intervention. It is recommended that health education that promotes the identification and control of modifiable risk factors, in particular, hypertension and healthy lifestyle modification among the populace. Early arrival to the hospital with a prompt and accurate institution of care, should emphasize intensive care for comatose patients with haemorrhagic stroke is essential in determining clinical outcome. This will, in no doubt, help in reducing the burden of stroke in Sub-Saharan African and in particular, Nigeria.

Authors' Contributions: KIE conceived and designed the study and did the literature review. Both authors participated in data collection and drafting of the manuscript.

Conflict of Interest: None.
Funding: Self-funded.

Publication History: Submitted 07 October 2018; Revised 20 December 2018; Accepted 11 February 2019.

\section{References}

1. WHO Monica Project, Principal Investigators. The World Health Organization MONICA Project (Monitory Trends and determinants in Cerebrovascular disease): a Major International Collaboration. J Clin Epidermiol 1988; 41: 105-114.

2. Sacco RL, Kasner SE, Broderick JP, Caplan LR, Connors JJ, Culebras A, et al. An updated definition of stroke for the 21st century. Stroke 2013; 44: 2064-2089.

3. Warlow CP, Dennis MS, Van Gijn J, Hankey GT, Sandercock PAG, Bamford JM, et al. Stroke: a practical guide to Management. Oxford: Blackwell Science 1996: 491-493.

4. O Donnel MJ, Xavier D, Liu 1. Risk factors for ischemic and intracerebral hemorrhagic stroke in 22 countries (The Interstroke Study): A case control study. Lancet. 2010; 376: 112-113.

5. Chukwudelunzu FE, Okwu-Delunzu VU, Dizissah J. Knowledge of Stroke Signs and Symptoms among Residents in a Rural South Eastern Nigerian Community. J Stroke Cerebrovasc Dis 2018; 27: 3306-3310.

6. Aho K, Harmsen P, Hatano S, Marquadsen J, Smirnov V, Strasser T. CVD in the community: results of $\mathrm{WHO}$ collaborative study. Bull World Health Org. 1980; 58: 113130.

7. Ogun SA, Oluwole O, Fatade B, Ogunseyinde AO, Ojini FI, Odusote KA. Comparison of the Siriraj Stroke Score and the WHO criteria in the clinical classification of stroke subtypes. Afr J Med Sci 2002; 31: 13-16. 
8. Nor AM, Davis J, Sen B, Shipsey D, Louw SJ, Dyker AG, et al. The Recognition of Stroke in the Emergency Room (ROSIER) Scale development and validation of a stroke recognition instrument. Lancet Neurol 2005: 4(11): 727-734.

9. Ricc S, Celani MG, Reghetti E. Clinical Methods for diagnostic confirmation of stroke Subtypes. Neuroepidermiol 1994; 13: 290-295.

10. Ogun S.A. Acute Stroke Mortality at Lagos University Teaching Hospital - a five year review. Niger Q J Hosp Med 2000; 10: 8-10.

11. Abubakar SA, Sabir AA. Profile of Stroke Patients seen in a tertiary health care center in Nigeria. Ann Niger Med 2013; 7: 55-59.

12. Ogun SA, Ojini F.I, Ogungbo B, Kolapo KO, Danesi MA. Stroke in South West Nigeria. Stroke 2005; 36(6): 1120-1122.

13. Obembe AO, Olaogun MO, Bamikole AA, Komalafe MA, Odetunde MO. Awareness of risk factors and warning signs of stroke in a Nigerian University. J Stroke Cerebrovasc. Dis 2014; 23(4): 749-758.

14. Desalu OO, Wahab KW, Fawale B, Olarenwaju TO, Busari OA, Adekoya AO, et al. A review of stroke admissions at a tertiary hospital in rural South Western Nigeria. Ann Afr Med 2011; 10: 80-85.

15. Njoku CH, Aduloju AB. Stroke in Sokoto, Nigeria: A five year retrospective study. Ann Afr Med 2004; 3: 73-76.

16. Ukoha OB, Ajaegbu O, Eke CO. A review of stroke cases in a military Hospital in Nigeria. Afrimedic J 2012; 2: 30-33.

17. Imarhiagbe FA, Ogbeide E, Ogunrin AO. Adult Stroke Registry in West Africa. Profile of 334 in-patients in the University of Benin Teaching Hospital, Benin City, Nigeria. Sahel Med J 2016; 19: 12-15.
18. Ojo O, Onyegiri CV. Stroke admissions in Kubwa General Hospital. A 30 month review. Sahel Med 2017; 20: 155-159.

19. Sacco RL, Benjamin EJ, Broderick JP, Dyken M, Easton JD, Feinberg WM, et al. American Heart Association Prevention Conference, IV: Prevention and rehabilitation of stroke: risk factors. Stroke.1997; 28: 1507-1517.

20. Sennay AG, Hannah SY. Types, risk profiles, and outcomes of stroke patients in a tertiary teaching hospital in Northern Ethiopia. eNeurologicalSci 2016; 3: 41-47.

21. Garbusinki JM, Vander Sande MA. Bartholome EJ, Dramaix M, Gaye A, Coleman $\mathrm{R}$, et al. Stroke presentation and outcome in developing countries. A prospective study in the Gambia. Stroke 2005; 36: 1388-1393.

22. Rosman KD. The epidemiology of stroke in an Urban Black Population. Stroke 1986; 17: 667-669.

23. Philip MW, Kenndy RL. ABC of arterial and venous disease. BMJ 2000; 320: 920-923.

24. Hansagi H, Romelsj OA, Gerhardsson de Verdier M, Andreasson S, Leifman A. Alcohol consumption and stroke mortality, a 20 year follow up of 15,077 men and women.. Stroke 1995;26: 1968-1973.

25. Bamford J, Dennis M, Sanderock P, Burn J, Warlow C. The frequency, causes and timing of death within 30 days of a first stroke. The Oxfordshire community stroke project 1981 - 1986. J Neurol Neurosurg Psychiatry 1990; 53: 967-971.

26. Bamford J, Sanderock P, Dennis M, Burn J, Warlow C. A prospective study of acute cerebrovascular disease in the community: the Oxford shire community stroke project 1981-1986.2. Incidence, case fatality rates and overall outcome at one year of cerebral infarction, primary intracerebral and 
subarachnoid hemorrhage. J Neurol Neurosurg Psychiatry 1990; 13: 16 - 22.

27. Venables G. Prognosis, outcome and recurrence of stroke. Stroke series 2002; 9: 103-105.
28. Ojini FI, Ogun SA, Danesi MA. Thirty days care fatality of stroke at LUTH. Niger Q J Hosp Med 2004; 14: 64-66.

29. Wahab KW. The burden of stroke in Nigeria. Int J Stroke 2008; 3: 290-292. 